\title{
The Role of PKC and HIF-1 and the Effect of Traditional Chinese Medicinal Compounds on Cerebral Ischemia-Reperfusion Injury
}

\author{
Zheyu Fang $\mathbb{D}$, ${ }^{1}$ Yangyang Zhang $\mathbb{D},^{2}$ Xixi Zhao $\mathbb{D}^{2},{ }^{2}$ Weifeng Jin $\mathbb{D},{ }^{3}$ and Li Yu $\mathbb{D}^{4}$ \\ ${ }^{1}$ The First School of Clinical Medicine, Zhejiang Chinese Medical University, Hangzhou 310053, China \\ ${ }^{2}$ School of Basic Medical Sciences, Zhejiang Chinese Medical University, Hangzhou 310053, China \\ ${ }^{3}$ School of Pharmaceutical Sciences, Zhejiang Chinese Medical University, Hangzhou 310053, China \\ ${ }^{4}$ School of Life Sciences, Zhejiang Chinese Medical University, Hangzhou 310053, China \\ Correspondence should be addressed to Li Yu; yuli9119@126.com
}

Received 3 November 2021; Revised 26 January 2022; Accepted 7 February 2022; Published 27 February 2022

Academic Editor: Mingbo Zhang

Copyright $\odot 2022$ Zheyu Fang et al. This is an open access article distributed under the Creative Commons Attribution License, which permits unrestricted use, distribution, and reproduction in any medium, provided the original work is properly cited.

\begin{abstract}
Neuronal death occurs during cerebral ischemia. However, when hemoperfusion and oxygen supply are resumed to the ischemic focus of the brain tissue, the brain tissue damage is further aggravated, resulting in cerebral ischemia-reperfusion injury (CIRI) to the patients. Protein kinase C (PKC) plays an important role in CIRI. Through the IP3/DAG/Ca ${ }^{2+}$ signaling pathway, it promotes the influx of calcium ions in neurons and causes calcium overload, which aggravates the damage. At the same time, when brain cells are hypoxic, hypoxia-inducible factor-1 (HIF-1) is expressed, which regulates the expression of Bcl2 and Bax through the PI3K/Akt signaling pathway and reduces nerve cell injury. It also fights hypoxic-ischemic injury by increasing the production of vascular endothelial growth factor (VEGF) to promote blood vessel formation. The PKC and HIF1 signaling pathways are also linked to CIRI. HIF-1 activates the PKC and ERK pathways via the upregulation of VEGF, leading to increased $\mathrm{Cx} 43$ phosphorylation and dysfunction and aggravating CIRI. Existing studies have shown that certain traditional Chinese medicine (TCM) compounds regulate the PKC and HIF-1 signaling pathways and alleviate CIRI. These compounds downregulate the PKC and the activity of the PKC-related signaling pathways to alleviate CIRI. They can also promote the expression of HIF-1, increase the content of VEGF in ischemic tissues to promote the generation of blood vessels, and improve microcirculation. TCM compounds can inhibit the cascade of reactions underlying disease occurrence and development by targeting multiple components using different herbal formulations to improve the structural and material changes in the brain cells, which alleviate CIRI and protect the brain tissue. This study briefly describes the role of PKC and HIF-1, their relationship in CIRI, and the effect of TCM on them.
\end{abstract}

\section{Introduction}

Stroke is a cerebrovascular disease caused by sudden rupture or blockage of blood vessels in the brain preventing blood from flowing into the brain and contributing to blood circulation disorders in the brain and brain tissue damage [1]. It is one of the three most deadly diseases in the world. Clinical cerebral apoplexy refers to the injury caused by an abnormal blood supply. Of these, $67.3 \sim 80.5 \%$ cases are that of ischemic cerebral apoplexy (also known as ischemic cerebrovascular disease) and the rest are hemorrhagic cerebral apoplexy. Studies have shown that severe cerebral ischemia can lead to irreversible infarction of nerve cells in the ischemic center. If there is a blood circulation disorder in the brain tissue, when the circulation and oxygen supply to the ischemic lesions of the brain are restored, the brain tissue will be more seriously damaged, resulting in a secondary cerebral ischemia-reperfusion injury (CIRI) [2]. This suggests that the treatment of cerebral ischemia should not only restore blood supply but also prevent secondary injury. Therefore, the role of a series of pathological and biochemical reactions caused by CIRI leading to neuronal damage and the effective protection of the brain tissue have always been an important topic for modern cerebrovascular disease research. Cerebral ischemia promotes the release of excitatory neurotransmitters, and excessive excitation leads 
to a large influx of $\mathrm{Ca}^{2+}$ in neurons, resulting in injury and apoptosis of nerve cells. It is noteworthy that in the mechanism of ischemia-reperfusion injury, the activation of PKC plays an important role in regulating the massive increase in intracellular $\mathrm{Ca}^{2+}$, leading to calcium overload [3]. At the same time, the expression of HIF-1 can promote the production of various hypoxia stress proteins [3]. However, PKC signal transduction has a special relationship with the HIF-1 signaling pathway and its factors in CIRI.

Ancient Chinese medicine books describe the treatment of cerebral ischemia using traditional Chinese medicine (TCM). According to the dialectical theory of qi and blood in TCM, cerebral ischemia is mainly caused by the imbalance of qi in the viscera, leading to "blood stasis." Therefore, activating blood circulation and alleviating blood stasis has become an important method in treating cerebral ischemia using TCM. In recent years, identifying methods for the prevention and treatment of cerebral ischemia using TCM has become a research hotspot due to the side effects of Western medicine. The combination therapy of various TCMs may provide a multitargeted intervention for the treatment of CIRI. Preliminary studies have shown that certain TCM compounds can treat CIRI with significant efficacy by regulating the PKC and HIF-1 signaling pathways.

In summary, in the context of CIRI, the role and interaction of PKC and HIF-1, as well as the effect of TCM on them, are important topics discussed in this article.

\section{Structure and Function of PKC}

Protein kinase C (PKC), a serine/threonine protein kinase, was discovered in the cytoplasm of rat neurons in 1977 by Nishizuka, a Japanese scholar. At least 12 subtypes of PKC have been found, and different subtypes of PKC have different physical and chemical properties and immune functions [4]. PKC is ubiquitous in several vertebrates and invertebrates and plays an important role in cell signal transduction pathways. There are three types of PKC based on differences in calcium and diglycerol dependence. The structures of these types are shown in Figure 1.

(1) Classical conventional PKC (cPKC): due to the specific characteristics of the regulatory region, $\mathrm{CPKC}$ is dependent on phosphatidylserine (PS), diacylglycerol (DAG), and calcium ions. The subtypes of $\mathrm{CPKC}$ can be divided into $\alpha, \beta \mathrm{I}, \beta \mathrm{II}, \gamma$, etc. (2) Novel PKC (nPKC): $\mathrm{nPKC}$ is regulated only by DAG. The subtypes of $\mathrm{nPKC}$ include $\delta, \varepsilon$, $\theta$, and $\eta$. (3) Atypical PKC (aPKC): aPKC cannot be activated by calcium ions and DAG but only by PS, and its subtype include $\iota, \lambda, \zeta$, and $\mu$. There is a high degree of structural homology across the different PKC subtypes, and all have the same catalytic region but different regulatory regions [5].

The conserved region $(\mathrm{C})$ and the variable region $(\mathrm{V})$ are two basic structural regions of cPKC. Based on the function, the $\mathrm{cPKC}$ structure can be divided into $\mathrm{N}$-amino terminal regulatory regions $(\mathrm{C} 1$ and $\mathrm{C} 2)$ and $\mathrm{C}$-carboxyl terminal catalytic regions (C3 and $\mathrm{C} 4)$ [6]. The regulatory region mainly regulates the activity of $\mathrm{PKC}$. The $\mathrm{C} 1$ region has two "zinc finger-like regions" responsible for binding DAG or phorbol esters [7]. Acidic lipids can bind to corresponding molecular sites in the $\mathrm{C} 2$ region, mediating binding to calcium ions. Therefore, PS, DAG, and calcium ions determine the activity of cPKC. The V 3 zone is called the hinge zone because it is responsible for connecting the regulatory and catalytic zones. The catalytic region is mainly responsible for substrate phosphorylation, the $\mathrm{C} 3$ region is mainly responsible for binding ATP [8], and the catalytic center is located in the $\mathrm{C} 4$ region.

The structure of $\mathrm{nPKC}$ is the same as that of $\mathrm{CPKC}$, but since there is no $\mathrm{C} 2$ region in the regulatory region, it cannot be activated by $\mathrm{Ca}^{2+}$, and its activity is only regulated by DAG [6]. aPKC differs from the other two mainly in the C1 and $\mathrm{C} 2$ regions. The $\mathrm{C} 1$ region of aPKC contains only one cysteine-rich region, so aPKC lacks sensitivity to DAG. At the same time, since aPKC does not contain a $\mathrm{C} 2$ region, its activity is regulated not only by $\mathrm{Ca}^{2+}$ but also by PS [6].

PKC, also known as the third messenger of cells, is involved in several pathophysiological processes in the central nervous system [7]. PKC functions mainly through the phosphorylation of intracellular proteins, which leads to a series of physiological reactions, including gene expression, cell degeneration and proliferation, synaptic plasticity, neurotransmitter release, and programmed cell death.

\section{Relationship between PKC and CIRI}

The mechanism underlying neuronal damage caused by PKC during CIRI is mainly the theory of translocation and activation of PKC [8]. In the resting state, PKC is distributed in the cytoplasm. When stimulated by upstream signaling molecules, PKC enters the nucleus, membrane, and organelle membranes, is activated, and participates in physiological and pathological processes.

3.1. PKC Signaling Pathway. The translocation and activation of PKC can promote the aggregation of intracellular $\mathrm{Ca}^{2+}$ to cause vasoconstriction in the brain tissue, which aggravates hypoxia and ischemia. The IP3/DAG/Ca ${ }^{2+}$ signaling pathway is the main pathway of $\mathrm{Ca}^{2+}$ aggregation induced by PKC [9]: the activation of $\mathrm{G}$ protein requires the interaction of signal molecules on the outside of the membrane with the receptor of the $G$ protein, and the activated $G$ protein, in turn, activates the downstream phospholipase C (PLC). Phosphatidylinositol $\left(\mathrm{PIP}_{2}\right)$ is decomposed by the activated phospholipase $\mathrm{C}$, resulting in the formation of inositol triphosphate $\left(\mathrm{IP}_{3}\right)$ that promotes the release of $\mathrm{Ca}^{2+}$ and diacylglycerol (DAG) that activates PKC. $\mathrm{Ca}^{2+}$ is released from the endoplasmic reticulum in large quantities and binds to calmodulin in the cytoplasm to participate in subsequent physiological and pathological processes. Under the coordination of $\mathrm{Ca}^{2+}$, the cascade of reaction caused by activated PKC reduces the permeability of cell membrane to calcium, promotes the massive influx of $\mathrm{Ca}^{2+}$ outside the membrane, aggravates calcium overload, and leads to the disorder of brain cell structure and functional metabolism. The above processes are shown in Figure 2. 

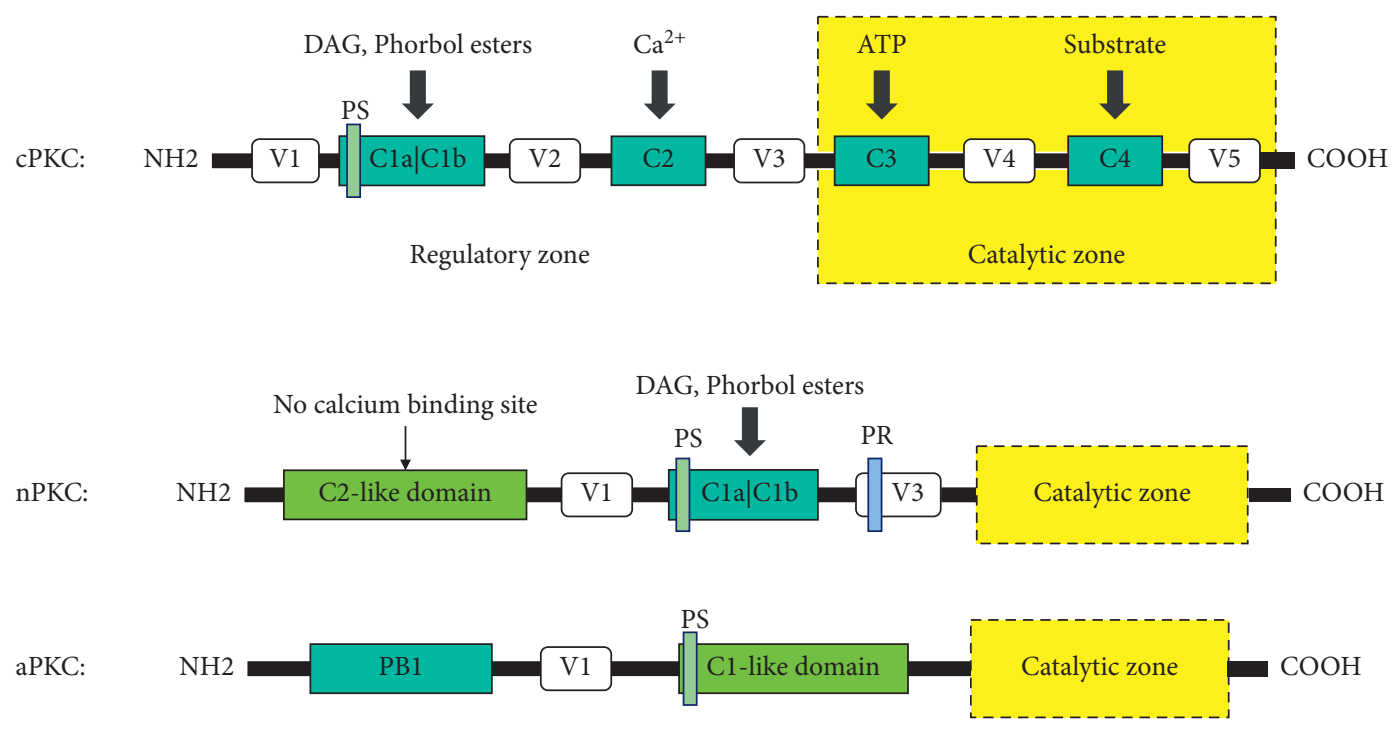

Figure 1: Schematic diagram of the PKC structure.

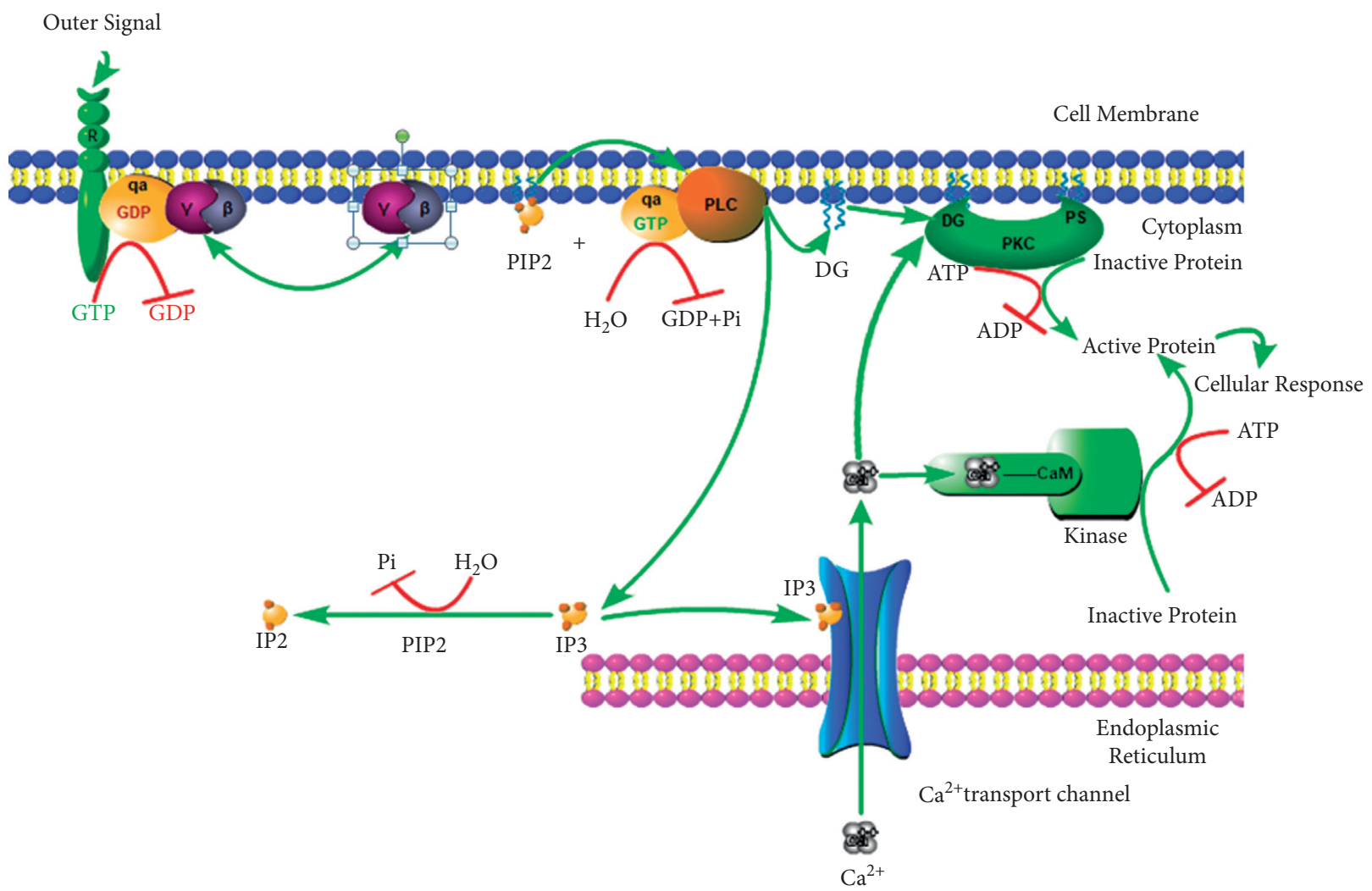

FIGURe 2: PKC signaling pathway.

3.2. Roles of PKC in CIRI. In addition to the above relationship, the following factors of CIRI are currently identified as being related to PKC.

3.2.1. PKC and Neurotransmitters. Neurotransmitter release is regulated by PKC, and it, thus, acts on CIRI. In CIRI, cerebral ischemia leads to changes in cell bioelectricity, and the pathological slow wave is aggravated and sustained due to the secondary injury caused by reperfusion. Inhibitory amino acids (inhibitory neurotransmitters), such as alanine, glycine, taurine, and $\gamma$-aminobutyric acid, were significantly elevated in early CIRI [10]. The excitatory amino acids, such as glutamic acid and aspartic acid, decreased significantly after ischemia-reperfusion and more significantly with time [11]. Through the regulation of PKC, glutamate release 
increases during CIRI, while the reuptake by neuronal cells decreases, resulting in extracellular glutamate accumulation, neuronal toxicity, and brain tissue damage.

3.2.2. PKC and Neuronal Receptors. PKC activates neuronal receptors, which accelerate brain damage [12]. Taking excitatory amino acids as an example, n-methyl-D-aspartate (NMDA) receptor, $\alpha$-amino-3-hydroxymethyl-propionic acid (AMPA) receptor, and metabotropic receptor are three subtypes of excitatory amino acid receptors. The activation of these three subtypes is related to PKC regulation. For example, PKC can phosphorylate postsynaptic NMDA receptors, thereby activating them to excitatory neurotransmitters [13]. The AMPA receptor activated by PKC can enhance its affinity to glutamate, thus promoting the opening of $\mathrm{Na}^{2+}$ channels and the depolarization of neurons. At the same time, large amounts of water enter cells, causing acute swelling of neurons and accelerating brain damage.

3.2.3. PKC and Protein Channels. Protein phosphorylation is an important method of protein activation [14], especially the activation of membrane receptors and channel proteins, and protein phosphorylation is inseparable from the regulation of PKC. Acid-sensing ion channels (ASICs), which sense the extracellular acidic environment, are highly expressed in the central nervous system [15], and their main functional subunit ASICs- $\alpha$ plays an important mediating role in the neuronal cell injury caused by cerebral ischemia. PKC regulates the activation of ASICs- $\alpha$ by phosphorylation of the NF- $\kappa \mathrm{B}$ signaling pathway molecules and promotes the expression of ASICs- $\alpha$, thereby enhancing cell damage induced by cerebral ischemia [16].

3.3. Roles of PKCIsoenzymes in CIRI. Current studies suggest that multiple PKC isoenzymes may play roles in CIRI.

3.3.1. PKC $-\gamma$ in CIRI. PKC- $\gamma$ exists only in the brain and spinal cord. It not only plays an important role in cell proliferation and differentiation but also mediates the release of neurotransmitters and the occurrence of tumors. PKC- $\gamma$ is dependent on $\mathrm{Ca}^{2+}$ and sensitive to phorbol ester or DAG. The increase in intracellular calcium ions stimulates the translocation of PKC- $\gamma$ to a certain extent, which is associated with the increase in intracellular PKC- $\gamma$ expression after ischemia-reperfusion. As a protective response of cells against injury [17], the increase in the PKC- $\gamma$ concentration increases the membrane content. If the PKC$\gamma$ gene is removed, its neuroprotective ability is weakened, making it vulnerable to ischemic injury, indicating that PKC- $\gamma$ has a protective effect on neurons. This was confirmed in the mouse middle cerebral artery occlusion experiment conducted by Aronowski et al. [18].

3.3.2. PKC- $\delta$ in CIRI. The activation of PKC- $\delta$ is independent of calcium ions, and PKC- $\delta$ widely exists in various tissues and cells. PKC- $\delta$ plays an important role in intracellular signal transduction pathways induced by various extracellular stimuli [19] and is an important mediator of CIRI. The activation of the NMDA receptor induces PKC- $\delta$ mRNA and protein expression during cerebral ischemia [12]. Studies found that PKC- $\delta$ induces CIRI by mediating an inflammatory response in PKC- $\delta$-knockout mice. The transplantation of bone marrow from PKC- $\delta$-knockout mice into wild mice alleviated ischemic injury [20], suggesting that $\mathrm{PKC}-\delta$ promotes perfusion injury. Meanwhile, PKC- $\delta$ is also associated with apoptosis in the delayed phase of cerebral ischemia-reperfusion and is an important therapeutic target in stroke patients during the long time window.

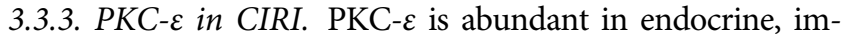
mune, and nerve cells and plays a role in cell proliferation and differentiation, gene expression, and certain other processes, covering a wide range. The activation of PKC- $\varepsilon$ is also independent of calcium ions but sensitive to phorbol ester and DAG. A large number of studies have shown that PKC- $\varepsilon$ is activated by adenosine through the signal transduction pathway [21] and regulates mitochondrial ATP-sensitive potassium channels, thus protecting neuronal cells from ischemic injury.

\section{Structure and Function of HIF-1}

Hypoxia-inducible factor-1 (HIF-1), which was first discovered by Semenza and Wang in 1992, can be detected in almost all human cells and is expressed stably in an anoxic environment. HIF-1 belongs to a class of heterologous protein dimers consisting of $\alpha$ and $\beta$ subunits. The major common domains of HIF-1 are as follows [22]: (1) the bHLH domain, or the $\mathrm{N}$-amino terminal, can bind to the DNA; (2) the PAS domain, or the intermediate region, is a key structural region for heterodimerization; and (3) C-carboxyl region, where proteins can bind to cofactors to regulate transcription.

Of the two subunits of HIF-1, only the $\alpha$ subunit is functional, namely, HIF- $1 \alpha$. It senses the hypoxic signals at two terminal regions. An oxygen-dependent degradation (ODD) domain is located at the C-carboxyl region, which plays a variety of roles, including regulating transcription and stabilizing hypoxia-inducible proteins. Trans-activation domain (TAD) is present at both ends, and TAD-N plays a necessary role in activating transcription, while TAD-C has a fine regulation function. In terms of structure, HIF- $1 \beta$ is similar to the HIF- $1 \alpha$ subunit, and its main function is to stabilize HIF-1 and promote conformational transition. In addition, the release of the $\alpha$ subunit is based on the binding of the $\beta$ subunit to form a heterodimer. The structure of HIF1 is shown in Figure 3.

It has been found that HIF- $1 \alpha$ can effectively inhibit apoptosis under mild hypoxia. However, if cells are exposed to severe hypoxia for a long time, the overexpression of HIF$1 \alpha$ can induce apoptosis [23].

\section{The Relationship between HIF-1 and CIRI}

HIF-1 has been shown to regulate more than 100 downstream target genes, most of which are related to energy metabolism or oxygen supply, such as EPO, VEGF, GLUT, 


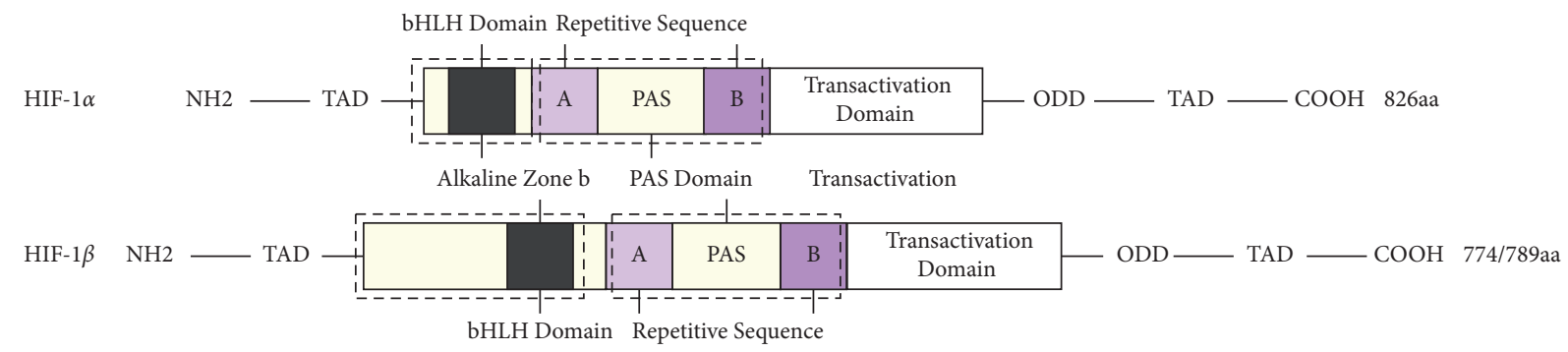

FIgURE 3: Schematic diagram of HIF-1 structure.

and apoptosis-related genes. When the body is hypoxic, HIF-1 specifically binds to the hypoxic-response elements contained in these genes. HIF-1 uses the protein products expressed by target genes to activate the body, including promoting the formation of blood vessels, upregulating the expression of red blood cells, increasing proliferation and differentiation of cells, regulating anaerobic metabolism, and decreasing apoptosis [24]. Thus, oxygen homeostasis in the hypoxic tissue can be maintained to counter the cascade of reactions caused by hypoxic ischemia.

By studying the animal model of focal CIRI, it was found that HIF- $1 \alpha$ and VEGF levels were upregulated in the ischemic penumbra, which contributed to the formation of blood vessels to resist CIRI [25]. The survival of neurons in the ischemic brain tissue depends largely on the stability of HIF- $1 \alpha$. HIF- $1 \alpha$ can effectively inhibit apoptosis, improve the survival rate of neuronal cells in mild hypoxia, and show a protective effect on the brain tissue [26].

$\mathrm{Bcl}-2$ and Bax can regulate cell apoptosis; the former can inhibit cell apoptosis, but the latter promotes cell apoptosis. In the hypoxic state, the conformation of Bax changes and it enters the mitochondrial outer membrane, thus affecting mitochondrial permeability. At the same time, cytochrome $\mathrm{C}$, generated by the mitochondria, is released from the transformation pore, enters the cytoplasm and binds caspase- 9 and APAF- 1 to form the apoptotic complex, activating the precursor caspase- 9 [27]. Activated caspase-9 continues to activate the downstream precursor caspase-3, which can cleave several different protein substrates, resulting in apoptosis. Cyto $\mathrm{C}$ can not only activate caspase- 3 but also produce a large number of free radicals via the peroxidation reaction, which blocks the generation of ATP in the mitochondria and impairs the function of mitochondria. Bcl-2 can bind Bax to form a heterodimer, which leads to the failure of mitochondrial permeability, thus inhibiting the generation of Cyto $C$ in the mitochondria, weakening the caspase cascade amplification effect, and inhibiting apoptosis [28].

When brain tissue is under hypoxic environment, a large number of activated HIF- $1 \alpha$ can upregulate the expression of $\mathrm{Bcl}-2$ through the PI3K/Akt pathway, while the expression of Bax pro-apoptotic protein and NF-KB is downregulated, promoting the increase in the ratio of Bcl-2/Bax and effectively inhibiting the cascade amplification effect of caspase-3. At the same time, HIF- $1 \alpha$ also effectively regulates caspase- 3 activity via VEGF, TNF- $\alpha$ pathway, and ERK1/2 signaling pathway [29], thus weakening the cascade amplification effect of caspase and reducing nerve cell damage. In addition, HIF- $1 \alpha$ also protects the damaged brain tissue by releasing antioxidants.

\section{Association between PKC and HIF-1 in CIRI}

Currently, there are only a few studies on PKC signal transduction and HIF-1 expression, especially on their association in CIRI. Certain studies targeting the most widespread intercellular protein, connexin 43 (Cx43), have shown that although $\mathrm{Cx} 43$ levels are significantly reduced in neurons in the damaged brain tissue, Cx40 levels are abnormally upregulated [30]. In addition, studies have found that the application of nonspecific gap junction blockers, such as 1-heptanol, can not only alleviate brain injury but also downregulate the expression of $\mathrm{Cx} 43$ [31], indicating that $\mathrm{Cx} 43$ may be one of the factors for the aggravation of brain tissue injury. Several studies have shown that HIF- $1 \alpha$ can decrease Cx43 function, but the relationship between $\mathrm{Cx} 43$ phosphorylation and brain injury has not been clarified. Recent studies have shown that $\mathrm{Cx} 43$ initiates the abnormal remodeling of the $\mathrm{Cx}$ family of proteins in the central nervous system, so the abnormal expression of Cx proteins may be closely related to CIRI. Studies have also confirmed that $\mathrm{Cx} 43$ plays an important role in the maintenance of cell morphology, suggesting that $\mathrm{Cx} 43$ has an effective cell protection effect [31]. Elbadawy found that increased $\mathrm{Cx} 43$ phosphorylation specifically inhibited $\mathrm{Cx} 43$ function, thereby enhancing the expression of HIF-1 $\alpha$ [32].

An increased level of activated HIF-1 $\alpha$ upregulates VEGF expression [33], which activates the PKC and ERK pathways [34], leading to more extensive Cx43 phosphorylation. Ultimately, it worsens the dysfunction of brain tissues. The downregulation of HIF- $1 \alpha$ expression in the hyperacute phase significantly reduced the phosphorylation of $\mathrm{Cx} 43$ protein and the expression of inflammatory factors in the hippocampus [35]. In conclusion, the downregulation of HIF- $1 \alpha$ expression can inhibit Cx43 phosphorylation, thus inhibiting the secretion of inflammatory factors by the nerve cells. This regulatory effect plays a protective role against brain injury. It is also suggested that the PKC and HIF-1 signaling pathways have a certain association in CIRI. The association between PKC and HIF-1 in CIRI is shown in Figure 4. 


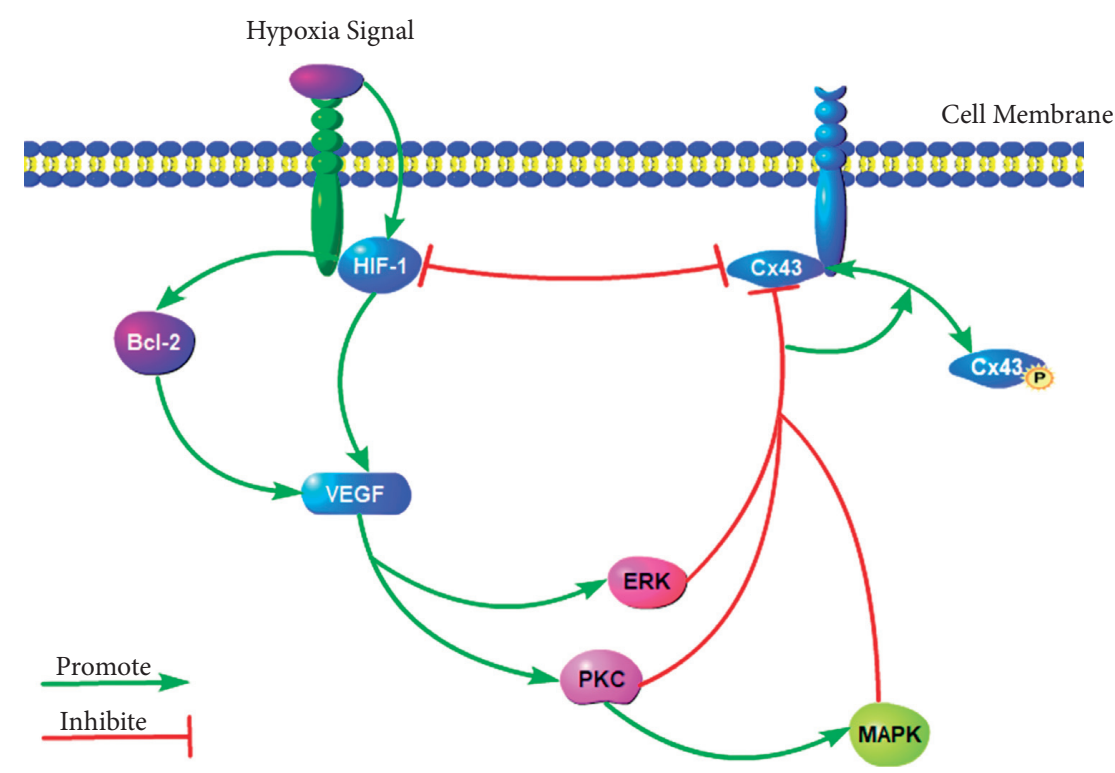

Figure 4: Association between PKC and HIF-1 in CIRI.

\section{The Effects of TCM Compounds on PKC and HIF-1}

A few studies conducted on the treatment of CIRI caused by cerebral ischemia, stroke, cerebral embolism, and other diseases by TCM compounds found that the therapeutic effect of TCM compounds is remarkable. Among these medicinal compounds, a few TCM components mainly act on the PKC and HIF-1 signaling pathways to achieve the therapeutic effect on CIRI.

\subsection{TCM Formulation Alleviates CIRI by Regulating the PKC Signaling Pathway}

7.1.1. Danhong Injection. Danhong injection is mainly extracted from Salvia miltiorrhiza and safflower and promotes blood circulation, alleviates blood stasis, and clears arteries [36]. It is widely used in the clinical treatment of cardiovascular and cerebrovascular diseases, especially in CIRI. PKC, an important signaling molecule in the development of CIRI, is essentially a multifunctional cytoplasmic enzyme. In the presence of a second messenger, PKC binds to the cell membrane, and cytoplasmic enzymes are activated to participate in various biochemical reactions and in the regulation of gene expression [37]. After the activation of PKC, cerebral vasoconstriction continues, resulting in CIRI [38]. Danhong injection significantly decreased the serum PKC content in patients. When Danhong injection was combined with butylphthalide, not only did the PKC content reduce significantly, but vasospasm was alleviated [39], suggesting that butylphthalide enhanced the efficacy of the Danhong injection.

Danhong injection is a commonly used compound preparation for the clinical treatment of CIRI since it inhibits the activity of $\mathrm{PKC}$ and the signaling pathway responsible for the occurrence and development of CIRI, to protect the brain cells. Butylphthalide can reduce the injury caused by cerebral ischemia by relieving the spasm and dilating the blood vessels. The efficacy and safety of Danhong injection were improved by combining butylphthalide, providing a novel drug using idea for the treatment of CIRI.

7.1.2. Qi Leech Capsule. Qizhi capsule is composed of Astragalus membranaceus, leeches, Salvia miltiorrhiza, Pheretima, and ephedra, etc. and mainly plays the role of invigorating qi, alleviating blood stasis, activating blood circulation, and clearing collaterals, and is an effective drug for the treatment of cerebral ischemia [40]. The treatment of cerebral ischemia by Qizhi capsule mainly includes two aspects: one is to the inhibition of CIRI by tanshinone IIA in astragalus [41] and the other is regulating the activity of the $\mathrm{Ca}^{2+}$-PKC-MARCKS signaling pathway and the phosphorylation of related proteins [42]. Excessive $\mathrm{Ca}^{2+}$ activates PKC in large quantities, which in turn phosphorylates MARCKS proteins to produce a large number of P-MARCKS. Highly expressed P-MARCKS activates microglia in the ischemic areas [43], which damages the neurons and their function. In addition, another component of the Qizhi capsule, hirudin extracted from the leech, can also protect brain cells [44], reduce cerebral vascular thrombosis, increase brain blood supply, and alleviate ischemia-reperfusion injury.

Qizhi capsule can inhibit the phosphorylation of PKC, MARCKS, and other proteins and downregulate the overexpression of P-PKC and P-MARCKS proteins, thus blocking the PKC-MARCKS signaling pathway and alleviating CIRI. The components of the Qizhi capsule can effectively scavenge free radicals and reduce the damage due to lipid peroxidation to the vascular endothelium. It can also inhibit thrombin activity, reduce thrombosis, reduce vascular permeability, and protect the vascular endothelium and brain cells. 
7.1.3. Buyang Huanwu Decoction. Buyang Huanwu decoction is composed of Astragalus membranaceus, Angelica sinensis, Ligusticum chuanxiong, Radix paeoniae, peach kernel, safflower, and earthworm, which has the effects of invigorating qi, promoting blood circulation, and clearing collaterals. It is a famous Chinese herbal formula for treating CIRI. In CIRI, vascular endothelial cells (VECs) are severely damaged, but they play an important role in preventing the occurrence of nonadaptive coagulation [45]. The thrombin receptor can cleave phosphoinositol to produce $\mathrm{IP}_{3}$ and DAG. DAG activates PKC and other $\mathrm{Ca}^{2+}$-sensitive proteins. Therefore, it is an important pathway for thrombin to induce VEC activation through PKC. It was found that Buyang Huanwu decoction plays an antithrombotic role by inhibiting the activation of PKC induced by thrombin [46, 47], and the effective ingredients, alkaloids and glycosides, are responsible for the inhibiting effect.

Buyang Huanwu decoction can not only improve the activity of fibrinolytic enzyme but also inhibit platelet aggregation and enhance the activity of anticoagulants, making it effective for the treatment of deficiency of qi and blood stasis. The active ingredients of Buyang Huanwu decoction have a significant effect on the treatment of CIRI-induced thrombosis and endothelial cell injury. PKC plays an important role in thrombosis induced by thrombin, indicating that Buyang Huanwu decoction can downregulate the expression of $\mathrm{PKC}$ and inhibit the PKC signaling pathway activated by thrombin, thus playing an antithrombotic role.

7.1.4. Ixeris sonchifolia Hance Injection. The Ixeris sonchifolia Hance injection is mainly extracted from Ixeris sonchifolia Hance, and has a complex chemical composition, including flavonoids, adenosine, triterpenoid saponins, and sesquiterpenoid lactones [48]. It removes heat and detoxifies the body, alleviates blood stasis, relieves pain, reduces swelling, helps in the drainage of pus, and is widely used to treat cardiovascular and cerebrovascular diseases [49]. The Ixeris sonchifolia Hance injection can inhibit the Rho A/ ROK- $\alpha$ and PKC- $\delta /$ MARCKS signaling pathways in addition to scavenging free radicals, exerting antioxidant effects, and inhibiting calcium overload and inflammatory factors [50]. Among them, the Rho A/ROK- $\alpha$ signaling pathway is involved in various mechanisms in CIRI, such as vasospasm [51], thrombosis [52], oxidative stress [53], and the promotion of apoptosis [54], while the PKC- $\delta /$ MARCKS signaling pathway also promotes CIRI. A study found that there was an interaction between the two pathways [55] and ROK$\alpha$ can promote the activation of PKC- $\delta$, which phosphorylates MARCKS and damages the brain cells. The Ixeris sonchifolia Hance injection can reduce the expression of the ROK- $\alpha$ protein and mRNA, inhibit the phosphorylation of PKC- $\delta$ and MARCKS, and play a protective role in cerebral ischemia. Studies have found that the PKC/MARCKS signaling pathway can induce hypoxia injury in cerebrovascular endothelial cells, but the Ixeris sonchifolia Hance injection can reduce the expression of P-PKC and P-MARCKS to inhibit the PKC/MARCKS signaling pathway and protect the vascular endothelial cells $[56,57]$.
The protective effect of the Ixeris sonchifolia Hance injection on hypoxic cells is bifold. On the one hand, the ROK$\alpha$ expression was decreased by downregulating the activities of the Rho A/ROK- $\alpha$ and PKC- $\delta /$ MARCKS signal pathways, and the phosphorylation of PKC- $\delta$ and MARCKS was decreased by the interaction, thus alleviating cerebral ischemia cell damage. On the other hand, it inhibits the PKC/ MARCKS signaling pathway directly to protect the hypoxic cells.

\subsection{TCM Formulation Alleviates CIRI by Regulating the HIF-1 Signaling Pathway}

7.2.1. Compound Danshen Tablet and Danhong Injection. The main ingredient of the compound Danshen tablet is Salvia miltiorrhiza, which has demonstrated a beneficial clinical effect on CIRI and promotes blood circulation and removes stasis, regulating qi and relieving pain. The active component of Danshen is mainly tanshinone, which can promote the high expression of HIF- $\alpha$ during cerebral ischemia $[58,59]$ and the generation of blood vessels in ischemic tissues, and improve microcirculation through the HIF-1/VEGF signaling pathway [60]. At the same time, the transcription of a series of genes downstream of HIF-1 is activated to increase the secretion of erythropoietin [61], induce angiogenesis, repair the damaged vascular endothelium, etc. In addition, the expression of GLUT1 and glycolytic enzymes also increases [62], to exert the antioxidant effect after reperfusion and improve brain tissue energy metabolism to increase oxygen supply to the brain tissue, improve microcirculation, and reduce brain damage. The main components of Danhong injection, Danshen and safflower, can activate the PI3K/AKT signaling pathway [36], upregulate the activities of $\mathrm{P}-\mathrm{AKT}$ and HIF- $1 \alpha$ proteins, decrease the expression of caspase-3 [63], reduce the apoptosis of nerve cells, and protect brain tissue.

In the ischemic brain tissue, both compound Danshen tablet and Danhong injection can act on the HIF-1 pathway to maintain the normal function of brain tissue by promoting increased expression of HIF- $1 \alpha$ to initiate the expression of downstream genes, thus increasing the generation of collateral circulation vessels to improve the hypoxia tolerance of nerve cells and reduce apoptosis.

7.2.2. Longzhi Decoction. Longzhi decoction inherited the function of Buyang Huanwu decoction to promote blood circulation and dissipate blood stasis, and the increased herbs, cyathula root, and leeches further enhanced the function of alleviating blood stasis and facilitating menstruation [64], making it an effective Chinese medicine preparation for the treatment of acute stroke. A study found that the treatment of Longzhi decoction can significantly improve the level of HIF-1 [65] and the content of downstream VEGF [66]. VEGF is a downstream gene activated by HIF- $1 \alpha$. It can promote microvascular formation through FIk-1 receptor protein $[67,68]$, improve microcirculation of ischemic focus, and accelerate the recovery of neurological function in the cerebral ischemic tissue. As another 
representative angiogenic factor, Ang-2, which is specifically expressed by the endothelial cells, is significantly expressed in the ischemic brain tissue after treatment with Longzhi decoction. A large amount of Ang-2 can promote angiogenesis and nerve repair $[69,70]$, eliminate the effect of VEGF on vascular permeability of diseased tissues [71], and maintain the stability of neovascularization.

Longzhi decoction can affect the expression of VEGF and its receptor genes in tissues around the cerebral infarct area through the HIF- $1 \alpha$ signaling pathway. The increased expression of HIF- $1 \alpha$ promotes the expression of VEGF, which is beneficial to the formation of ischemic focal vessels. Longzhi decoction can also improve the expression of ANG2 , synergistic with HIF- $1 \alpha$ and VEGF, to promote angiogenesis in ischemic and its surrounding areas, protect vascular units, reduce the increase in vascular permeability induced by VEGF, and play a neuroprotective role.

7.2.3. Yangyin Tongnao Granule. Yangyin Tongnao granule is a TCM compound preparation mainly composed of Rehmannia glutinosa, Astragalus membranaceus, Ligusticum chuanxiong, and Pueraria root [72]. The main effective components in the compound are catalpol, astragaloside IV, ligustrazine, puerarin, and ferulic acid. The treatment effect of Yangyin Tongnao granule is demonstrated, and it shows the effects of dilating blood vessels, inhibiting thrombosis, and improving microcirculation and neuroprotection [73]. Experimental studies have shown that serum HIF- $1 \alpha$ and VEGF levels in acute cerebral ischemia-reperfusion rats can be significantly increased by the treatment of Yangyin Tongnao granule [74-76], indicating that the active components of Yangyin Tongnao granule have a good therapeutic effect in brain injury repair. Inflammation is closely involved in the occurrence and development of ischemiareperfusion injury [72, 77]. The intracytoplasmic pattern recognition receptor, NOD-like receptor (NLRP3), is activated when cells are infected and bind to pro-caspase- 1 to form the NLRP3 inflammasome. NLRP3 inflammasome activates pro-caspase- 1 to form caspase-1, which decomposes inflammatory mediators, such as IL- $1 \beta$ and IL-18 precursors, into inflammatory factors with important biological activity [78], participating in various inflammatory processes and aggravating CIRI. The treatment with Yangyin Tongnao granule can significantly reduce the content of NLRP3 inflammasome [79], and a large number of activated HIF- $1 \alpha$ can inhibit the formation of caspase- 1 through the PI3K/Akt/Bcl-2 pathway [28], blocking the occurrence of the above cascade process, thus playing a protective role in the ischemic brain tissue.

Yangyin Tongnao granules can increase the expression of HIF- $1 \alpha$ and VEGF in ischemic lesions, promote the formation of new blood vessels, relieve ischemia and hypoxia, and improve microcirculation due to the combined action of the main active ingredients of different TCMs. At the same time, it inhibits the activation of the NLRP3 inflammasome, reduces the formation of caspase-1 via different signal pathways, and decreases the inflammatory response of CIRI, which is conducive to the repair and functional recovery in the nerve tissue of the ischemic focus.

7.2.4. Ginsenoside. Ginsenosides are the main biologically active components of ginseng [80], which are categorized into Rg1-3, Rb1-2, and Rc types. These triterpene saponins have been shown to have many beneficial effects on the human body, especially on the nervous system. Ginsenosides can reduce excitotoxicity, oxidative stress, and neuroinflammation, maintain neurotransmitter balance, resist apoptosis, and maintain mitochondrial stability [81], which contribute to effectively reducing CIRI and protecting the nervous system $[82,83]$. Studies have shown that ginsenosides induce the large amounts of expression of HIF- $1 \alpha$ in large amounts through the HIF-1 $\alpha$ /VEGF signaling pathway $[84,85]$. As a downstream target gene of HIF-1, VEGF can be transcriptionally activated to enhance angiogenesis and reduce CIRI. At the same time, the high expression of HIF$1 \alpha$ and VEGF induced by ginsenosides can increase the proliferation and differentiation of neural stem cells in ischemic and hypoxic brain tissues [86, 87], promote the repair and generation of nerves, and help the recovery of brain function.

Ginsenoside regulates the transcription of the HIF- $1 \alpha$ gene and initiates the transcription of the downstream target gene VEGF, which can not only promote collateral circulation via the formation of new blood vessels but also improve the ischemic and hypoxic state of microcirculation. At the same time, it acts on neural stem cells to promote their proliferation and differentiation to repair brain damage and restore brain function.

7.3. TCM Formulation Alleviates CIRI by Regulating the PKC/ HIF-1 Signaling Pathway. Guhong injection (GHI) is a compound preparation composed of safflower extract (a TCM) and acetylglutamine (Western medicine) [88]. It has anti-inflammatory and antioxidant effects; therefore, it is widely used in the treatment of cerebrovascular diseases [89]. Hypoxia is a key factor in the survival of cells injured by ischemia. Studies have shown that GHI can reduce the concentration of HIF- $1 \alpha, \mathrm{PKC}$, and EPO in the serum and inhibit the expression of HIF- $1 \alpha$ mRNA and protein in the brain [90]. These results indicated that GHI has a protective effect on CIRI by inhibiting the PKC/HIF- $1 \alpha$ signaling pathway. Nicotinamide adenine dinucleotide phosphate oxidase 4 (NOX4) is the main oxidative enzyme in the pathogenesis of cerebral ischemia [91]. PKC plays a major role in the NADPH oxidase signaling pathway, and in the MAPK and NF-KB signaling pathways [92]. In prolonged cerebral ischemia, the level of prolyl hydroxylase 2 (PHD2) decreases, probably because PHD2 is the transcription target of HIF- $1 \alpha$ [90]. When the expression of HIF- $1 \alpha$ decreases, PHD2 also decreases. PKC inhibits the activation of PHD2, while HIF- $1 \alpha$ induces the hydroxylation and degradation of PHD2 [93]. HIF- $1 \alpha$ protects gene transcription by regulating the adaptive response to hypoxia and other stresses and plays a key role in the activation of endogenous substances downstream of cerebral ischemia. Studies have shown that 
EPO and iNOS are the key downstream substances activated by HIF- $1 \alpha$ [94]. In addition, EPO is an inducer of ischemic tolerance and can prevent the death of apoptotic cells. Therefore, GHI upregulates the expression of EPO and iNOS in the brain of rats with cerebral ischemia injury.

$\mathrm{GHI}$ induces a protective effect on cerebral ischemia via the PKC/HIF- $1 \alpha$ signaling pathway, which may be a potential mechanism for GHI to alleviate CIRI. Therefore, in addition to treating ischemic stroke, GHI also has the potential to treat cerebrovascular diseases. As a combination of Chinese and Western medicine, GHI has more multitarget pharmacological effects. Therefore, other mechanisms underlying the protective effect of GHI on cerebral ischemia are worthy of further study.

\section{Problems and Prospects}

PKC and HIF-1 are highly expressed in cerebral ischemia and reperfusion, and there is a special relationship between the two signaling pathways and comprising factors. The interaction and mechanism between the two are of great importance to elucidate CIRI. The elucidation of the relationship between PKC and HIF-1 is expected to identify novel therapeutic targets, which is of great significance to the prognosis and treatment of CIRI. Based on the result of treatment of CIRI using TCM compounds targeting PKC and HIF-1, research on the new TCM compounds is needed to improve the therapeutic effect on CIRI.

Western medicines usually work via a single therapeutic target that is influenced by the patient's genotype and comorbidities. Because the metabolic rate of drugs varies greatly among different populations, there is the individualization of drugs. The treatment of TCM is usually by different compatibility of TCM compound. Compared to single-target Western medicine, TCM compound, which is a multicomponent and multitarget drug preparation, may have a more extensive clinical application.

As an important supplement or substitute for Western medicine, TCM preparations are widely used in clinical practice (mainly in China) to improve the clinical outcome of patients with cerebrovascular diseases. These protective mechanisms include the inhibition of oxidative stress and inflammation, anticoagulant and antithrombotic effects, reduction in apoptosis and blood pressure, dilation of blood vessels, and promotion of angiogenesis. Since most clinical trials are conducted in China and the sample size is limited, the efficacy and safety of TCM compounds still need to be further verified through high-quality, large-scale, multicenter, and randomized controlled clinical trials. In addition, due to the multicomponent nature of TCM compounds, the multitarget mechanism in cerebrovascular diseases is still not clear. Further molecular, cellular, and animal studies are needed to elucidate their multipharmacological characteristics and accelerate the overall development and utilization of TCM preparations.

The study of the combination of TCM and other Western medicine is an important direction, which may enhance the therapeutic effect of drug preparation. The emergence of systematic and network pharmacology will also provide new insights into the therapeutic potential of TCM preparations in cerebrovascular diseases. Therefore, the development and utilization of TCM compounds in cerebrovascular therapy have laid a good foundation for the development of TCM.

\section{Data Availability}

No data were used to support this study.

\section{Conflicts of Interest}

The authors have no conflicts of interest to declare.

\section{Authors' Contributions}

All the authors made a significant contribution to the work.

\section{Acknowledgments}

The authors have received funding for research, writing, and publication of this paper from the National Natural Science Foundation of China (Grant no. 81904083) and National College Student Innovation and Entrepreneurship Training Program (Grant no. S202110344026).

\section{References}

[1] H. Amani, E. Mostafavi, M. R. Alebouyeh et al., "Would colloidal gold nanocarriers present an effective diagnosis or treatment for ischemic stroke?" International Journal of Nanomedicine, vol. 14, pp. 8013-8031, 2019.

[2] S. Q. Li, J. Z. Wang, and L. H. Yin, Pathophysiology, People's Medical Publishing House, Beijing, China, 2013.

[3] T. S. Niu, G. X. Qi, and P. Fu, "The impact of ischemiapreconditioning on expression of hypoxia inducible factor-1a gene in acute myocardial infarction rats and the involvement of PKC signal pathway," Chinese Journal of Arteriosclerosis, vol. 16, no. 10, pp. 796-800, 2008.

[4] V. Akoyev, S. Das, and S. Jena, "A review of the preclinical and clinical evidence for protein kinase $\mathrm{C}$ as a largest for drug development for bipolar," Current Psychiatry Reports, vol. 10, no. 6, pp. 510-519, 2008.

[5] G. L. Ye, "Protein kinase C and ischemic brain damage," Foreign Medical Sciences Cerebrovascular Diseases, vol. 4, no. 5, pp. 266-269, 1996.

[6] C. M. Dowling, S. L. Hayes, J. J. Phelan et al., "Expression of protein kinase $\mathrm{C}$ gamma promotes cell migration in colon cancer," Oncotarget, vol. 8, no. 42, pp. 72096-72107, 2017.

[7] H. B. Li, X. J. Zhao, and J. L. Yin, "Role of protein kinase C signaling pathway in cerebral ischemia-reperfusion injury," Journal of Brain and Nervous Diseases, vol. 24, no. 2, pp. 120-122, 2016.

[8] D. Snell, K. R. Lord, T. B. Tabakof, and P. L. Hoffman, "Protea $\mathrm{k}$ masse $\mathrm{C}$ activation attenuates $\mathrm{N}$-methy L-D-A spartats-induoextm-CIYEases minlmcellular calcmmmcerebellar granule cells," Journal of Neurochemistry, vol. 62, no. 5, pp. 1783-1784, 1994.

[9] Y. E. Luo and Z. Yang, "Overview of the role of protein kinase $\mathrm{C}$ and inhibitors in cerebral ischemia/reperfusion injury," Journal of Tianjin College of Traditional Chinese Medicine, vol. 23, no. 3, pp. 167-168, 2004. 
[10] C. Tao, M. Y. Li, and P. Qu, "Mechanism of excitatory transmitter glutamate release during cerebral ischemiareperfusion injury," Science \& Technology Information, vol. 13, no. 24, pp. 244-245, 2015.

[11] Y. Nishizawa, "Glutamate release and neuronal damage in ischemia," Life Sciences, vol. 69, no. 4, pp. 369-381, 2001.

[12] H. Yang, X. M. Han, and J. Y. Xu, "Study on the expression of PKC $\delta$ after ischemia and reperfusion in rats and the protective effect of Ginaton," Chinese Journal of Gerontology, vol. 21, no. 4, pp. 285-287, 2001.

[13] P. C. Suen, K. Wu, and J. L. Xu, "NMDA receptor subunits in the postsynaptic density of rat brain: expression and phosphorylation by endogenous protein kinases," Molecular Brain Research, vol. 80, no. 3, pp. 1547-1551, 1988.

[14] S. G. Khasar, Y.-H. Lin, A. Martin et al., "A novel nociceptor signaling pathway revealed in protein kinase $\mathrm{C} \varepsilon$ mutant mice," Neuron, vol. 24, no. 1, pp. 253-260, 1999.

[15] D. K. Ways, C. A. Kukoly, J. deVente et al., "MCF-7 breast cancer cells transfected with protein kinase C-alpha exhibit altered expression of other protein kinase $\mathrm{C}$ isoforms and display a more aggressive neoplastic phenotype," Journal of Clinical Investigation, vol. 95, no. 4, pp. 1906-1915, 1995.

[16] L. Zhang, "Regulation of acid-sensing ion channel 1a (ASICla) protein expression and function by protein kinase C," Anhui Medical University, Hefei, China, Chinese Master's Theses Full-text Database, 2020.

[17] G. Yu, Y. Luo, and G. G. Peng, "Effect and mechanism of protein kinase C inhibitor on cerebral ischemia in rats," Stroke and Nervous Diseases, vol. 9, no. 6, pp. 321-324, 2002.

[18] J. Aronowski, J. C. Grotta, R. Strong, and M. N. Waxham, "Interplay between the gamma isoform of PKC and calcineurin in regulation of vulnerability to focal cerebral ischemia," Journal of Cerebral Blood Flow and Metabolism, vol. 20, no. 2, pp. 343-349, 2000.

[19] S. J. Lai and D. F. Chen, "Research progress on the role of PKC $\delta$ and related signaling pathways in NASH," International Journal of Digestive Diseases, vol. 33, no. 2, pp. 82-85, 2013.

[20] W.-H. Chou, D.-S. Choi, H. Zhang et al., "Neutrophil protein kinase $\mathrm{C} \delta$ as a mediator of stroke-reperfusion injury," Journal of Clinical Investigation, vol. 114, no. 1, pp. 49-56, 2004.

[21] N. Di-Capua, O. Sperling, and E. Zoref-Shani, "Protein kinase $\mathrm{C}-\varepsilon$ is involved in the adenosine-activated signal transduction pathway conferring protection against ischemia-reperfusion injury in primary rat neuronal cultures," Journal of Neurochemistry, vol. 84, no. 2, pp. 409-412, 2003.

[22] F. John, O’Rourke, and Y.-M. Tian, "Pugh Oxygen-regulated and transactivating domains in endothelial PAS protein 1: comparison with hypoxia-inducible factor-1alpha," Biological Chemistry, vol. 274, no. 4, pp. 2060-2071, 1999.

[23] G. L. Semenza, "Hypoxia-inducible factor 1: oxygen homeostasis and disease pathophysiology," Trends in Molecular Medicine, vol. 7, no. 8, pp. 345-350, 2001.

[24] T. Toninaga, S. Kure, and K. Narisawa, "Evidence of apoptosis cell death after focal cerebral ischemia," Brain Research, vol. 624, no. 1, pp. 32-38, 1993.

[25] F. Shen, Y. Fan, H. Su et al., "Adeno-associated viral vectormediated hypoxia-regulated VEGF gene transfer promotes angiogenesis following focal cerebral ischemia in mice," Gene Therapy, vol. 15, no. 1, pp. 30-39, 2008.

[26] O. O. Ogunshola and A. Al-Ahmad, "HIF-1 at the blood-brain barrier: a mediator of permeability?" High Altitude Medicine \& Biology, vol. 13, no. 3, pp. 153-161, 2012.

[27] M. Haraguchi, S. Torii, S.-i. Matsuzawa et al., "Apoptotic protease activating factor 1 (Apaf-1)-Independent cell death suppression by bcl-2," Journal of Experimental Medicine, vol. 191, no. 10, pp. 1709-1720, 2000.

[28] J. Yang, S. Zhao, X. Yang, H. Zhang, P. Zheng, and H. Wu, "Inhibition of B-cell apoptosis is mediated through increased expression of Bcl-2 in patients with rheumatoid arthritis," International Journal of Rheumatic Diseases, vol. 19, no. 2, pp. 134-140, 2016.

[29] B.-N. Liu, B.-X. Han, and F. Liu, "Neuroprotective effect of pAkt and HIF-1 $\alpha$ on ischemia rats," Asian Pacific Journal of Tropical Medicine, vol. 7, no. 3, pp. 221-225, 2014.

[30] W. Chen, Y. Guo, W. Yang, P. Zheng, J. Zeng, and W. Tong, "Connexin40 correlates with oxidative stress in brains of traumatic brain injury rats," Restorative Neurology and Neuroscience, vol. 35, no. 2, pp. 217-224, 2017.

[31] K. Li, H. Zhou, L. Zhan et al., "Hypoxic preconditioning maintains GLT-1 against transient global cerebral ischemia through upregulating Cx43 and inhibiting c-src," Frontiers in Molecular Neuroscience, vol. 11, pp. 344-346, 2018.

[32] H. M. Elbadawy, P. Mirabelli, M. Xeroudaki et al., "Effect of connexin 43 inhibition by the mimetic peptide Gap27 on corneal wound healing, inflammation and neovascularization," British Journal of Pharmacology, vol. 173, no. 19, pp. 2880-2893, 2016.

[33] A. Zimna and M. Kurpisz, "Hypoxia-inducible factor-1 in physiological and pathophysiological angiogenesis: applications and therapies," BioMed Research International, vol. 2015, Article ID 549412, 13 pages, 2015.

[34] L. I. Da Wei, B. O. Le, and Q. Tu, "Prenatal hypoxia altered angiotensin II-mediated vasoconstrictions via PKC/ERK/ ROCK pathways and potassium channels in rat offspring middle cerebral artery," Biomedical and Environmental Sciences, vol. 34, no. 3, pp. 250-255, 2021.

[35] W. Chen, D. B. Ren, and P. Zhen, "Phosphorylation of Cx43 regulated by HIF- $1 \alpha$ in cerebral ischemia-reperfusion injury," Journal of Clinical Neurosurgery, vol. 16, no. 2, pp. 142-146, 2019.

[36] C. Wan, Y Zhang, L Yu et al., "Neuroprotective effect of Danhong injection on cerebral ischemia-reperfusion injury in rats by activation of the PI3K-akt pathway," Frontiers in Pharmacology, vol. 11, p. 298, 2020.

[37] W. C. Shyu, S. Z. Lin, and P. S. Yen, "Stromal cell-derived factor-1 alpha promotes neuroprotection, angiogenesis, and mobilization/homing of bone marrow-derived cells in stroke rats," Journal of Pharmacology and Experimental Therapeutics, vol. 324, no. 2, pp. 834-849, 2018.

[38] C. Michiels, "Endothelial cell functions," Journal of Cellular Physiology, vol. 196, no. 3, pp. 430-443, 2003.

[39] B. C. Xiao and S. X. Peng, "Effect of butylphthalide combined with Danhong injection in the treatment of acute cerebral infarction and its influence on PKC, CRP and PTX3," International Journal of Laboratory Medicine, vol. 41, no. 9, pp. 1109-1111+1115, 2020.

[40] T. T. Zhang, R. Teng, and X. J. Lu, "Effects of Qizhi Capsule on expression of protein by PKC-MARCKS signaling pathway in ischemic stroke model rats," China Journal of Traditional Chinese Medicine and Pharmacy, vol. 36, no. 1, pp. 402-406, 2021.

[41] R. Wang, J. T. Sun, and Y. He, "The efficacies of tanshinone IIA for the treatment of ischemic cerebral stroke and its economic evaluation," Chinese Journal of Neuromedicine, vol. 6, no. 9, pp. 967-969, 2007.

[42] B. P. Ziemba, J. E. Burke, G. Masson, R. L. Williams, and J. J. Falke, "Regulation of PI3K by PKC and MARCKS: single-molecule 
analysis of a reconstituted signaling pathway," Biophysical Journal, vol. 110, no. 8, pp. 1811-1825, 2016.

[43] Q. H. Zhang, Y. L. Zhang, and J. L. Lou, "Changes of MARCKS mRNA expression in rat hippocampus with acute multi-cerebral infarction," Chinese Journal of Pathophysiology, vol. 22, no. 5, pp. 964-967, 2006.

[44] H. B. Jiang, Y. G. Zhu, and J. Z. Liu, "Study on the anticoagulation effect of hirudin," Hebei Journal of Traditional Chinese Medicine, vol. 33, no. 8, pp. 1236-1237, 2011.

[45] J. Ahamed and W. Ruf, "Protease-activated receptor 2-dependent phosphorylation of the tissue factor cytoplasmic domain," Journal of Biological Chemistry, vol. 279, no. 22, pp. 23038-23044, 2004.

[46] M. E. Ou, L. W. Tang, and C. Q. Deng, "Effect of Buyang Huanwu decoction and its active fraction on antithrombosis in cultured vascular endothelial cells and protein kinase C," Chinese Traditional and Herbal Drugs, vol. 39, no. 10, pp. 1514-1520, 2008.

[47] J. Ruf and R. Wolfram, "Protease-activated receptor 2-dependent phosphorylation of the tissue factor cytoplasmic domain," Journal of Biological Chemistry, vol. 279, no. 22, pp. 23038-23044, 2004.

[48] X. Liao, G. Q. Hua, and Y. M. Xie, "Systematic report on reevaluating Kudiezi injection," China Journal of Chinese Materia Medica, vol. 39, no. 18, pp. 3626-3629, 2014.

[49] L. P. Fang, Z. C. Xiao, and H. Y. Ke, "Impact of Kudiezi injection on hemorheology of recovery phase patients with cerebral infarction," Practical Journal of Cardiac Cerebral Pneumal and Vascular Disease, vol. 24, no. 3, pp. 93-94, 2016.

[50] X. C. Han, J. T. Zhou, and L. Li, “Advances in the mechanism of Kudiezi in the treatment of central nervous system diseases," Chinese Journal of Modern Applied Pharmacy, vol. 36, no. 17, pp. 2222-2227, 2019.

[51] S. Chrissobolis and C. G. Sobey, "Recent evidence for an involvement of Rho-kinase in cerebral vascular disease," Stroke, vol. 37, no. 8, pp. 2174-2180, 2006.

[52] T. Nakakuki, M. Ito, H. Iwasaki et al., "Rho/Rho-kinase pathway contributes to C-reactive protein-induced plasminogen activator inhibitor-1 expression in endothelial cells," Arteriosclerosis, Thrombosis, and Vascular Biology, vol. 25, no. 10, pp. 2088-2093, 2005.

[53] B. A. Diebold, B. Fowler, J. Lu, M. C. Dinauer, and G. M. Bokoch, "Antagonistic cross-talk between Rac and Cdc42 GTPases regulates generation of reactive oxygen species," Journal of Biological Chemistry, vol. 279, no. 27, pp. 28136-28142, 2004.

[54] J. Shi and L. Wei, "Rho kinase in the regulation of cell death and survival," Archivum Immunologiae et Therapiae Experimentalis, vol. 55, no. 2, pp. 61-75, 2007.

[55] W. Bai, "Regulation of the interaction between ROK $\alpha$ and PKC $\delta / M A R C K S$ signaling pathway in rats with acute cerebral ischemia by detoxication and collateralization," Chinese Doctoral Dissertations Full-text Database, Beijing University of Chinese Medicine, Beijing, China, 2010.

[56] J. Y. Yang, J. L. Yu, and Z. Dai, "Regulation effect of Kudiezi Injection on PKC $\delta / M A R C K S$ signal pathway in vascular endothelial cells after hypoxia injury," Journal of Beijing University of Traditional Chinese Medicine, vol. 40, no. 3, pp. 214-218, 2017.

[57] J. L. Yu, "Protection of hypoxic-injured vascular endothelial cells and the regulation of PKC $\delta / M A R C K S$ pathway," Beijing University of Chinese Medicine, Beijing, China, Chinese Master's Theses Full-Text Database, 2013.
[58] S. X. Xin, "The effect of tanshinone IIa on the expression of HIF-1 alpha gene in hypoxic ischemic brain damage newborn rat brain," Zhejiang Chinese Medical University, Hangzhou, China, Chinese Master's Theses Full-Text Database, 2014.

[59] C. Chen and L. L. Wang, "Tanshinone IIA has effect on gene expression of hypoxia-inducible factor- $1 \alpha$ in neonatal rats with hypoxic-ischemic brain damage," Journal of New Chinese Medicine, vol. 50, no. 12, pp. 16-19, 2018.

[60] L. Z. Li, J. J. Liu, and X. Y. Li, "Effect of Danhong injection on vascular endothelial growth factor in rats with focal cerebral ischemia reperfusion injury," Practical Pharmacy and Clinical Remedies, vol. 18, no. 08, pp. 897-900, 2015.

[61] H. Zhu, L. Zou, J. Tian, G. Du, and Y. Gao, "SMND-309, a novel derivative of salvianolic acid $\mathrm{B}$, protects rat brains ischemia and reperfusion injury by targeting the JAK2/STAT3 pathway," European Journal of Pharmacology, vol. 714, no. 1-3, pp. 23-31, 2013.

[62] X. X. Wang, J. Wang, and H. B. Tong, "Tanshinone IIA ameliorates neuronal damage in ischemic stroke model by targeting GLUT1: a mechanistic study," Chinese Remedies and Clinics, vol. 20, no. 18, pp. 2988-2993, 2020.

[63] C. H. Yuan, M. X. Chen, and Y. Li, "Impacts of salvia miltiorrhiza on p-AKT, Caspase-3 and HIF- $1 \alpha$ protein in brain tissue of cerebral ischemia-reperfusion injury," China Medicine and Pharmacy, vol. 11, no. 9, pp. 23-26, 2021.

[64] Y. S. Zhang, Y. Z. Ma, and Z. P. Qian, "Clinical observation on treatment of acute cerebral infarction with qi deficiency and blood stasis syndrome by Buyang Huanwu Decoction," Acta Medicinae Sinica, vol. 32, no. 3, pp. 92-95, 2019.

[65] R. Feng, Q. H. Liu, and Y. B. Chen, "Research on proangiogenetic effect and mechanism of Longzhi decoctionon on qi deficiency and blood stasis in rats with acute cerebral infarction," Liaoning Journal of Traditional Chinese Medicine, vol. 44, no. 5, pp. 1084-1087+1120, 2017.

[66] G. L. Liang, "Clinical efficacy and mechanism of action of Longzhi decoction in treatment of acute cerebral infarction with qi deficiency and blood stasis," Journal of Hunan University of Chinese Medicine, vol. 38, no. 10, pp. 1191-1194, 2018.

[67] W. Jiang, "The infection of Longzhi Tang to the Vegf and FLK1Mrna expression of the focal cerebral ischemia rat models' peri-infarction tissue," Guangxi Medical University, Nanning, China, Chinese Master's Theses Full-text Database, 2014.

[68] Y. C. Li and H. Wang, "Relationship between vascular endothelial growth factor and HIBD," Foreign Medical Sciences Section of Psychiatry, vol. 32, no. 4, pp. 210-213, 2005.

[69] L. Marteau, S. Valable, D. Divoux et al., "Angiopoietin-2 is vasoprotective in the acute phase of cerebral ischemia," Journal of Cerebral Blood Flow and Metabolism, vol. 33, no. 3, pp. 389-395, 2013.

[70] A. Androutsellis-Theotokis, M. A. Rueger, D. M. Park et al., "Angiogenic factors stimulate growth of adult neural stem cells," PLoS One, vol. 5, no. 2, Article ID e9414, 2010.

[71] S. W. Lee, W. J. Kim, H. O. Jun, Y. K. Choi, and K. W. Kim, "Angiopoietin-1 reduces vascular endothelial growth factorinduced brain endothelial permeability via upregulation of ZO-2," International Journal of Molecular Medicine, vol. 23, pp. 279-284, 2009.

[72] Y. Wang, J. H. Yang, and Y. He, "Yangyin Tongnao Granule exerts the neuroprotective effects and inhibiting NF- $\kappa \mathrm{B}$ signaling pathway in cerebral ischemia/reperfusion injury rats," China Journal of Traditional Chinese Medicine and Pharmacy, vol. 32, no. 8, pp. 3713-3717, 2017. 
[73] H. T. Wan, X. D. Bie, and Z. Yao, "Treating ischemic stroke patients of deficiency of qi and yin syndrome and static blood obstructing collaterals syndrome by Yangyin yiqi huoxue recipe: a clinical study of therapeutic effect," Chinese Journal of Integrated Traditional and Western Medicine, vol. 35, no. 3, pp. 281-286, 2015.

[74] H. F. Zhou, Y. W. Tan, and R. B. Yang, "Combined pharmacodynamics and pharmacokinetic in vivo study on active ingredients of Yangyin Tongnao Granules in rats with focal cerebral ischemia," China Journal of Traditional Chinese Medicine and Pharmacy, vol. 33, no. 9, pp. 4061-4065, 2018.

[75] Y. Wang, J. Yang, H. Du, H. Zhang, H. Wan, and Y. He, "Yangyin Tongnao granules enhance neurogenesis in the periinfarct area and upregulate brain-derived neurotrophic factor and vascular endothelial growth factor after focal cerebral ischemic infarction in rats," Molecular Biology Reports, vol. 46, no. 4, pp. 3817-3826, 2019.

[76] Z. W. Li, "Study on the effects of Buyang Huanwu decoction and its prescriptions on cerebral ischemia reperfusion injury and the mechanism of promoting angiogenesis," Zhejiang Chinese Medical University, Hangzhou, China, Chinese Master's Theses Full-text Database, 2020.

[77] P. Yin and X. W. Liu, "The expression and clinical implication of TLR3 in myocardial ischemia-reperfusion injury of rat model," Immunological Journal, vol. 30, no. 9, pp. 768-772+791, 2014.

[78] N. Zhang, X. Zhang, X. Liu et al., "Chrysophanol inhibits NALP3 inflammasome activation and ameliorates cerebral ischemia/reperfusion in mice," Mediators of Inflammation, vol. 2014, Article ID 370530, 12 pages, 2014.

[79] Y. Rong, Y. He, and L. Cheng, "Protection mechanism of the main active ingredients combination of Yangyin Tongnao granules on cerebral ischemia/reperfusion injury rats," Chinese Pharmaceutical Journal, vol. 53, no. 3, pp. 199-204, 2018.

[80] T. Ahmed, S. H. Raza, A. Maryam et al., "Ginsenoside Rb1 as a neuroprotective agent: a review," Brain Research Bulletin, vol. 125 , pp. 30-43, 2016.

[81] Z.-H. Jin, "Enhancement of oral bioavailability and immune response of Ginsenoside Rh2 by co-administration with piperine," Chinese Journal of Natural Medicines, vol. 16, no. 2, pp. 143-149, 2018.

[82] Y. H. Dong, T. T. Hu, and L. P. Dai, "Advances in pharmacological effects of Gensenoside Rg1 on the cardiovascular system and the nervous system," Chinese Journal of Ethnomedicine and Ethnopharmacy, vol. 25, no. 3, pp. 30-32, 2016.

[83] Y. Yu, G. Sun, Y. Luo et al., "Cardioprotective effects of Notoginsenoside R1 against ischemia/reperfusion injuries by regulating oxidative stress- and endoplasmic reticulum stressrelated signaling pathways," Scientific Reports, vol. 6, no. 1, p. 21730, 2016.

[84] Z. Z. Shi, Y. Bai, and J. W. Cheng, "Protective effects of ginsenosides on cerebral ischemia-reperfusion injury in rats by regulating HIF- $1 \alpha$-VEGF pathway," Journal of Sichuan of Traditional Chinese Medicine, vol. 37, no. 6, pp. 43-46, 2019.

[85] D. J. Wang, Q. Y. Li, and S. J. Xu, "Effect of ginsenoside Rg1 on angiogenesis after neonatal hypoxia ischemia brain damage in rats," Journal of Sichuan University (Medical Science Edition), vol. 42, no. 4, pp. 503-507, 2011.

[86] J. Gao, "Effects of ginsenosides on neural stem cells and the regulatory mechanism of HIF-1a-VEGF pathway," Beijing University of Chinese Medicine, Beijing, China, Chinese Master's Theses Full-text Database, 2018.
[87] H. J. Bai, Effects of Ginsenoside on the Proliferation and Differentiation of Neural Stem Cells after Stroke, Beijing University of Chinese Medicine, Beijing, China, 2015.

[88] H. Wang, H. Zhou, Y. He et al., "Protective effect of naoxintong capsule combined with Guhong injection on rat brain microvascular endothelial cells during cerebral ischemiareperfusion injury," Chinese Journal of Integrative Medicine, vol. 10, pp. 744-751, 2020.

[89] J. Ai, H. Wan, M. Shu et al., "Guhong injection protects against focal cerebral ischemia-reperfusion injury via antiinflammatory effects in rats," Archives of Pharmacal Research, vol. 40, no. 5, pp. 610-622, 2017.

[90] L. Yu, Y. Zhang, X. Zhao, H. Wan, Y. He, and W. Jin, “Guhong injection alleviates cerebral ischemia-reperfusion injury via the PKC/HIF- $1 \alpha$ pathway in rats," Frontiers in Pharmacology, vol. 12, Article ID 716121, 2021.

[91] J. B. Sun, Y. Li, Y. F. Cai et al., "Scutellarin protects oxygen/ glucose-deprived astrocytes and reduces focal cerebral ischemic injury," Neural regeneration research, vol. 13, pp. 1396-1407, 2018.

[92] K.-L. Tsai, T.-H. Chiu, M.-H. Tsai, H.-Y. Chen, and H.-C. Ou, "Vinorelbine-induced oxidative injury in human endothelial cells mediated by AMPK/PKC/NADPH/NF- $\kappa \mathrm{B}$ pathways," Cell Biochemistry and Biophysics, vol. 62, no. 3, pp. 467-479, 2012.

[93] B. F. Gibbs, I. M. Yasinska, A. E. Oniku, and V. V. Sumbayev, "Effects of stem cell factor on hypoxia-inducible factor 1 alpha accumulation in human acute myeloid leukaemia and LAD2 mast cells," PLoS One, vol. 6, no. 7, Article ID e22502, 2011.

[94] H. Wang, F. Niu, W. Fan, J. Shi, J. Zhang, and B. Li, "Modulating effects of preconditioning exercise in the expression of ET-1 and BNP via HIF- $1 \alpha$ in ischemically injured brain," Metabolic Brain Disease, vol. 34, no. 5, pp. 1299-1311, 2019. 